D. BOICHARD ${ }^{1}$, C. GROHS ${ }^{2}$, F. BOURGEOIS ${ }^{3}$,

F. CERQUEIRA ${ }^{3}$, R. FAUGERAS ${ }^{3}$, A. NEAU $^{4}$, D. MILAN ${ }^{5}$, R. RUPP ${ }^{1}, Y$ Y. AMIGUES ${ }^{3}$, M.-Y. BOSCHER ${ }^{3}$, H. LEVÉZIEL ${ }^{2}$.

1 INRA, Station de Génétique Quantitative et Appli quée, 78352 J ouy-en-J osas cedex

2 INRA, Laboratoi re de Généti queBi ochi mi que et de Cytogénéti que, 78352 J ouy-en-J osas cedex

${ }^{3}$ LABOGENA, Domai ne de Vilvert, 78352 J ouyen-J osas cedex

4 INRA, Département de Génétique Animale, 78352 J ouy-en-J osas cedex

5 INRA, Laboratoi re de Généti que Cellulai re, BP 27, 31326 Castanet-Tolosan cedex

e-mail : Di di er.Boi chard@dga.jouy.inra.fr
7 - Utilisation des marqueurs génétiques

\section{La recherche de QTL à l'aide de marqueurs : résultats chez les bovins laitiers}

R ésu mé. Un programme de détection de QTL est conduit dans les races bovi nes Prim'Holstein, Normandeet Montbéli arde Ce di sposi tif dit 'peti tes-filles' i mpli que 1554 taureaux d'i nsémi nati on artifi cielle, fi ls de 14 pères et indexés sur descendance pour 24 caractères de production lai ti ère, conformati on, ferti li té, rési stance aux mammi tes et faci li té de trai te Ces taureaux sont génotypés pour 169 marqueurs répartis sur le génome. La recherche de QTL est réali sée par régressi on li néai re intra père en prenant en compte toute l'i nformati on marqueurs par groupe de liaison. Des QTL sont détectés pour chaque caractère, y compris pour les caractères peu héri tables. L'effet de substi tuti on alléli que est souvent compris entre 0,6 et 1 écart type généti que. Une mi nori té de pères (entre 1 et 5 seulement sur 14) apparai ssent hétérozygotes pour chaque QTL identifié La locali sati on des QTL est peu préci se, l'i ntervalle de confi anceétant toujours supéri eur à $20 \mathrm{cM}$. Ce travai I se poursuit d'une part avec la mi seen place d'unesélecti on assi stée par marqueurs, d'autre part avec la caractéri sati on de certai ns QTL dans le cadre du programme européen Euri bdis.

Chez les bovins laitiers, deux programmes de détection de QTL sont conduits par le Département de Génétique Animale de I'INRA. Le premier, terminé et en cours d'analyse, est un dispositif intra race et ses caractéristiques et ses résultats sont présentés dans cet article. Le deuxième, en cours au domaine du Pin-au-Haras, est un dispositif de croisement F2 entre les races Prim'Holstein et Normande. II impliquera environ 600 femelles F 2 en première lactation entre 1999 et 2004.

Le programme de détection de QTL intra race est un dispositif 'petites-filles' utilisant la structure des populations nationales et des programmes de sélection existants. II est donc réalisé avec la collaboration technique et financière de la profession. D'autre part, il implique des taureaux d'insémination artificielle (IA) testés sur descendance, qui n'expriment donc pas les caractères étudiés et qui sont pour la plupart déjà morts. La finalité première d'un tel dispositif est donc la sélection assistée par marqueurs, des données complémentaires étant par ailleurs indispensables pour caractériser plus précisément les QTL.

\section{1 / Le dispositif 'petites-filles'}

La détection de QTL avec des marqueurs (Boichard et al 1998) repose sur la recherche d'une association entre la transmission d'un allèle du marqueur et le niveau de performance. Intra population, du fait de l'équilibre de liaison existant a priori entre marqueurs et QTL, cette recherche d'association doit impérativement être réalisée intra famille. Chez les bovins, le nombre de descendants par femelle est limité, de sorte que l'analyse ne peut être réalisée qu'intra famille de père. Dans le type de dispositif le plus simple, dit 'filles', on recherche une association entre l'allèle marqueur reçu du père par le produit et la performance de ce produit. Dans le dispositif 'petites-filles' (Weller et al 1990), les fils sont eux-mêmes parents et les données phénotypiques analysées sont les performances moyennes de leurs descendants (figure 1). Le protocole implique donc trois générations, les pères et leurs fils qui sont génotypés, leurs petitesfilles, qui ne sont pas génotypées, mais dont les performances sont mesurées. L'intérêt de ce dispositif est multiple :

- dès lors que l'effectif de petites-filles par fils est raisonnable, la variabilité résiduelle de la performance moyenne est réduite, ce qui induit un gain de puissance par rapport au dispositif 'filles', en dépit du fait que l'effet à estimer soit divisé par deux ;

- la puissance du dispositif est moins dépendante de l'héritabilité du caractère ;

- les mâles étant des taureaux d'IA, leur ADN est facilement disponible.

Les contraintes liées à ce dispositif sont tout aussi nombreuses :

- le nombre de mesures phénotypiques est extrêmement élevé, de sorte que ces données doivent préexister et il n'est généralement pas envisageable de rajouter de nouveaux caractères ;

- la structure des dispositifs est imposée par la structure de la population et un nombre limité de populations se prêtent à ce type d'analyse. C'est cependant le cas des grandes races bovines françaises dans lesquelles chaque taureau d'IA est évalué sur descendance et ces taureaux sont issus d'un nombre limité de pères, constituant ainsi d'assez grandes familles de demi-frères (Boichard et al 1996). 
Figure 1. Principe du dispositif 'petites-filles'.

Un QTL a deux allèles $Q$ et $q$, l'effet de substitution de $q$ par $Q$ est de a. Le taux de recombinaison entre le $Q T L$ et le marqueur $M$ est de $r$. Un grand-père est supposé double hétérozygote $M Q / m q$ au marqueur et au $Q T L$. Ses fils se répartissent en quatre catégories selon l'haplotype reçu du père, $M Q$ et mq avec une probabilité (1r)/2, $m Q$ et $M q$ avec une probabilité r/2. Si le fils a reçu $Q$ de son père, la moitié des nf petites-filles reçoivent $Q$, l'autre moitié recevant un allèle grand-maternel $x$, et la supériorité moyenne de la descendance est a/4. La différence entre les deux groupes de descendance des fils ayant reçu $Q$ et $q$ est donc de a/2. La variabilité résiduelle de la moyenne des petites-filles se décompose en une variabilité entre familles de petites-filles intra grandpère $\left(3 V_{g} / 16\right)$ et une variabilité résiduelle intra famille divisée par l'effectif de petites-filles $\left(\left(1-h^{2} / 4\right) V_{p} / n f\right)$. Bien que l'effet du QTL soit deux fois plus faible que dans un protocole «filles », le protocole est généralement plus puissant car la composante résiduelle est réduite dans une proportion plus élevée, et ce d'autant plus que nf est élevé et que la variabilité génétique est faible.

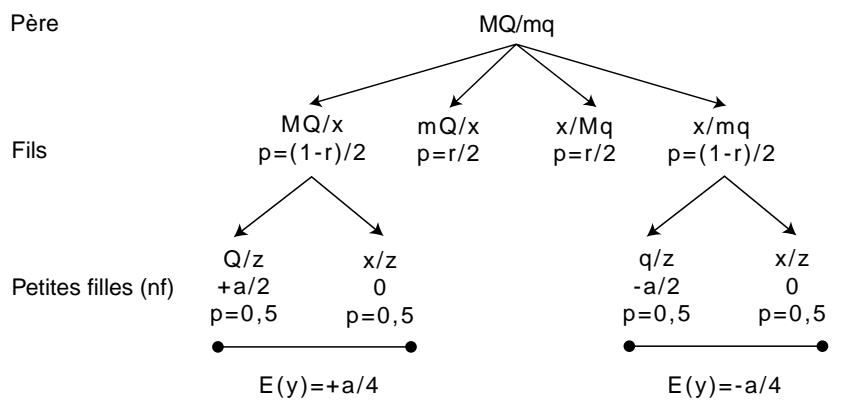

\section{2 / Matériel animal}

Le dispositif mis en place comprend les 14 familles décrites au tableau 1 , dont 9 en race Holstein, 3 en race Normande et 2 en race Montbéliarde. Ces familles ont chacune un impact important dans leur race respective. L'effectif total de fils est de 1554, soit une moyenne de 111 par famille ; leur naissance est relativement récente : entre 1988 et 1992. La taille des familles varie de 59 à 232.

Tableau 1. Familles analysées.

\begin{tabular}{|l|l|}
\hline RACE & \multicolumn{1}{|c|}{ PÈ RES } \\
\hline Holstein & Blackstar, Ugela Bell, Leadman, \\
& Micheal, Cleitus, Secret, Ned Boy, \\
& Southwind, Jesse \\
Normande & Talbot, Sahara, Rivage \\
Montbéliarde & Tibet, Tartars \\
\hline
\end{tabular}

Pour disposer de l'ADN de l'ensemble des taureaux impliqués dans le dispositif, une banque de semence a été mise en place à I'INRA en 1993 ; elle est alimentée chaque année par les unités de sélection volontaires, qui envoient 25 à 50 doses de leurs taureaux mis en testage. A ce jour, elle comprend des doses de plus de 8500 taureaux laitiers, avec un excellent niveau d'exhaustivité pour les cohortes nées depuis 1986.
Ces taureaux ont été évalués sur descendance, avec 80 filles en moyenne, pour 24 caractères de production : quantités de lait (lait), de matière grasse $(\mathrm{mg})$, de matière protéique ( $\mathrm{mp})$, taux butyreux (tb) et taux protéique (tp), de résistance aux mammites (cell), de fertilité femelle (fert), de facilité de traite (vtr), de conformation de la mamelle : profondeur du sillon (psil), distance plancher-jarret (dplj), équilibre (eqaa), écart latéral (ecla), implantation (impa), écart avant (ecav), longueur des trayons (Igtr), attache avant (atta) et hauteur de l'attache arrière (hata), de format : hauteur au sacrum (hsac) et profondeur de poitrine (ppoi), et de caractères relatifs au bassin : longueur (loba), largeur (laba) et inclinaison (inba) ainsi qu'aux membres: angle du jarret (anja) et épaisseur du talon (epta).

\section{3 / Marquage moléculaire}

Les marqueurs génétiques sont de deux types. D'une part, l'information disponible en routine pour tous les taureaux d'insémination artificielle est bien sûr prise en compte. II s'agit des 11 groupes sanguins utilisés en contrôle de filiation ainsi que du gène BLAD. Par ailleurs, un réseau de 157 marqueurs microsatellites a été choisi sur la base des trois critères suivants :

- informativité des marqueurs : un marqueur choisi doit, sauf exception, être à l'état hétérozygote chez au moins 8 des 14 pères;

- localisation chromosomique des marqueurs : tous les chromosomes autosomaux doivent être couverts, avec une distance maximum entre marqueurs d'environ $20 \mathrm{~cm}$ (les chromosomes sexuels ont été négligés car tous les fils ont reçu le chromosome $Y$ de leur père et parce que sa partie pseudoautosomale est réduite et peu polymorphe) ;

- qualité technique des marqueurs : les marqueurs doivent être facilement amplifiables par PCR et interprétables sur un séquenceur d'ADN. Ces contraintes, et principalement la première, ont conduit à rejeter environ la moitié des 300 marqueurs testés au Laboratoire de Génétique Biochimique et de Cytogénétique. Les 29 autosomes sont couverts, avec un nombre de marqueurs par chromosome variant de 4 à 10.

L'ensemble des 1568 individus (14 pères et 1554 fils) ont été génotypés pour les 157 microsatellites choisis, ce qui représente environ 240000 typages. Les mères n'ont pas été génotypées. Les génotypages ont été réalisés au GIE LABOGE NA. Les marqueurs ont été assemblés par jeu de 8 à 12 microsatellites, amplifiés par PCR en 1 ou 2 multiplex, puis analysés sur séquenceur d'ADN. Chaque gel a été interprété indépendamment par les deux logiciels Gemma et Genotyper ${ }^{\circledR}(A B I)$. Les résultats validés ont ensuite été transférés dans la base de données Mapgena. Les typages se sont échelonnés entre juin 1996 et juin 1999.

\section{4 / Analyses statistiques}

Six produits présentant des résultats incompatibles avec ceux de leur père pour de nombreux marqueurs ont été éliminés, sans que l'on ait déterminé s'il s'agit d'erreur de paternité ou d'erreur de manipulation. Les analyses suivantes ont été conduites sur 1548 produits et 169 marqueurs, dont 157 microsatellites. Les caractéristiques des micro- 
satellites sont présentées dans le tableau 2. Les marqueurs sélectionnés sont très informatifs puisqu'en moyenne 10 pères sur 14 sont hétérozygotes, les deux tiers des génotypages sont informatifs (on déduit sans équivoque l'allèle paternel transmis). Leur polymorphisme est élevé puisque le nombre moyen d'allèles d'origine maternelle trouvés chez les fils est d'environ 9.

Tableau 2. Caractéristiques des microsatellites utilisés.

\begin{tabular}{|l|c|c|c|c|}
\hline & Moyenne & Ecart type & Min. & Max. \\
\hline Typages réalisés & 1526 & 20 & 1401 & 1546 \\
Pères hétérozygotes & 9,9 & 2,1 & 4 & 14 \\
Méioses informatives & 1007 & 188 & 472 & 1301 \\
Allèles chez les mères & 9,0 & 3,2 & 3 & 24 \\
PIC ${ }^{(1)}$ chez les mères & 0,635 & 0,134 & 0,290 & 0,857 \\
\hline
\end{tabular}

(1) Polymorphism Information Content (Lander and Botstein 1987)

Une carte génétique spécifique a été construite. Elle a permis d'une part de préciser les distances entre marqueurs à partir d'un dispositif de grande taille, d'autre part de cartographier certains marqueurs pour la première fois, comme le BLAD sur le chromosome 1 et le groupe sanguin EAF sur le chromosome 17. Elle couvre une longueur totale de 3205 cM Haldane, ou 2049 cM Kosambi (Vaiman 2000, cet ouvrage).

La recherche de QTL est réalisée en deux étapes selon la méthodologie proposée par Elsen et al (1999).

Après avoir estimé la probabilité des différentes phases possibles des chromosomes paternels à partir de l'information marqueur de leur descendance, on choisit la phase la plus probable et on estime pour chaque descendant la probabilité qu'il ait reçu l'un ou l'autre segment chromosomique de son père, et cela en tout point de son génome. L'estimation de cette probabilité prend en compte tous les marqueurs d'un même chromosome.

La détection de QTL proprement dite est réalisée chromosome par chromosome, à toutes les positions avec un pas de $1 \mathrm{cM}$, à l'aide d'un modèle de régression intra père.

$$
\mathrm{y}_{\mathrm{ij}}=\mathrm{s}_{\mathrm{i}}+\left(2 \mathrm{p}_{\mathrm{ij}}-1\right) \mathrm{a}_{\mathrm{i}}+\mathrm{e}_{\mathrm{ij}}
$$

La variable $y_{i j}$ est la performance moyenne des $n_{i j}$ filles du fils $j$ du père $i$, après correction pour divers effets indépendants du fils et estimés dans les procédures d'évaluation génétique. Pour chaque position x sur le génome, $s_{i}$ est l'effet du père $i, a_{i}$ est l'effet de l'allèle 1 du père $i$, (et - $a_{i}$ l'effet de l'allèle 2 du père), $p_{i j}$ est la probabilité que le fils $j$ ait reçu de son père i l'allèle 1 , conditionnellement à l'information marqueur, et $e_{i j}$ est la résiduelle du modèle, supposée de moyenne nulle et de variance hétérogène $s_{i}{ }^{2} / C D_{i j}$, le $C D$ étant la précision de l'index, fonction du nombre de petitesfilles par fils et de l'héritabilité du caractère. Pour améliorer la robustesse de la détection (Goffinet et al 1999) et pour analyser des caractères exprimés parfois sur des échelles différentes entre races, la variance résiduelle est définie intra famille. D'autre part, la prise en compte du CD de l'index du fils traduit le fait que le nombre de petites-filles varie entre fils et que plus il est faible, plus la variabilité résiduelle est importante (figure 1).
Un point particulièrement critique est le test de signification. La statistique de test calculée pour la position $x$ du génome dépend fortement de celle calculée au voisinage de $x$, de sorte que le nombre de tests indépendants n'est pas connu a priori. Une méthode pour contrôler le risque d'erreur et construire des seuils de rejet adaptés a été proposée par Churchill et Doerge (1994) : les performances sont permutées aléatoirement intra famille, alors que les données de marqueurs restent inchangées. Une éventuelle association entre marqueurs et performance est alors détruite, ce qui crée une situation sans QTL lié. L'analyse de cette situation fournit une valeur de la distribution de la statistique de test sous I'hypothèse qu'il n'y a pas de QTL en position x. La valeur maximum observée sur l'ensemble du chromosome fournit une valeur de la distribution de la statistique sous I'hypothèse qu'il n'y a pas de QTL sur le chromosome considéré (hypothèse $\mathrm{HO}$ ). Cette opération est répétée 10000 à 50000 fois pour obtenir une distribution empirique de la statistique de test sous HO. Le seuil correspondant à un risque $p$ donné ( $5 \%$ par exemple) est déduit du quantile correspondant dans la distribution empirique.

Toutefois, le nombre de tests réalisés reste toujours élevé ( 24 caractères $\times 29$ chromosomes = 696). Pour un risque de $5 \%$ par analyse (c'est-à-dire pour chaque combinaison caractère $x$ chromosome), on attend donc environ 35 faux positifs. Des seuils de rejet plus élevés peuvent être considérés, de façon à limiter le risque à un niveau q pour l'ensemble de l'expérience. Un risque q à l'échelle de l'expérience correspond à un risque $p$ pour chaque test tel que : $1-q=(1-p)^{n}$, n étant le nombre de tests indépendants réalisés. Cependant, la définition de seuils aussi élevés conduit à une perte de puissance considérable et donc à la non détection de QTL, ce phénomène étant paradoxalement d'autant plus marqué que le nombre de caractères analysés augmente. Une solution intermédiaire consiste à ne prendre en compte que la multiplicité des tests pour un caractère donné, soit le nombre de chromosomes (29). Pour une probabilité $q=10 \%$ et 24 caractères, on attend donc 2 ou 3 résultats faux positifs sur l'ensemble des tests. Ceci correspond à un risque par caractère et chromosome de $p=0,4 \%$.

Les résultats significatifs à 0,4 \% par caractère sont significatifs à l'échelle de l'expérience avec un haut degré de confiance. Les résultats significatifs à un risque inférieur à $1 \%$ par caractère et par chromosome sont eux aussi assez fiables puisqu'on n'attend que 6 faux positifs. Les résultats significatifs à un risque compris entre 1 et $4 \%$ sont aussi présentés succinctement, mais, compte tenu du nombre de faux positifs attendus (28), ils demandent à être confirmés.

\section{5 / Résultats}

Le tableau 3 montre les QTL détectés avec un risque inférieur à $0,4 \%$ à l'échelle du chromosome, ce qui correspond à des QTL quasi certains. Le tableau donne également le marqueur le plus proche de la position la plus probable du QTL, mais il est important de noter que la précision de la localisation est médiocre, toujours supérieure à 20 $\mathrm{cM}$ et souvent largement plus. 
Tableau 3. Liste des QTL détectés avec un risque inférieur à $0,4 \%$ (détection avec certitude). $n$ : le nombre de familles.

\begin{tabular}{|c|c|c|c|c|}
\hline Caractère & $\begin{array}{l}\text { Chromo } \\
\text {-some }\end{array}$ & $\begin{array}{l}\text { marqueur } \\
\text { proche }\end{array}$ & $\begin{array}{c}\text { risque } \\
(\%)\end{array}$ & $\mathrm{n}$ \\
\hline lait & 14 & CSSM 66 & 0,07 & 4 \\
\hline $\mathrm{mg}$ & $\begin{array}{l}14 \\
19 \\
26\end{array}$ & $\begin{array}{c}\text { CSSM66 } \\
\text { ETH3 } \\
\text { IDVGA59 }\end{array}$ & $\begin{array}{l}0,16 \\
0,13 \\
0,01\end{array}$ & $\begin{array}{l}3 \\
4 \\
6\end{array}$ \\
\hline $\mathrm{mp}$ & 26 & IDVGA59 & 0,04 & 3 \\
\hline $\mathrm{tb}$ & 14 & CSSM66 & 0,01 & 5 \\
\hline tp & $\begin{array}{c}6 \\
14\end{array}$ & $\begin{array}{l}\text { INRAK } \\
\text { CSSM 66 }\end{array}$ & $\begin{array}{l}0,04 \\
0,01\end{array}$ & $\begin{array}{l}3 \\
3\end{array}$ \\
\hline cell & 15 & BMS2684 & 0,05 & 5 \\
\hline psil & 28 & BMS2060 & 0,27 & 5 \\
\hline eqaa & $\begin{array}{l}18 \\
19\end{array}$ & $\begin{array}{l}\text { IDVGA55 } \\
\text { HEL } 10\end{array}$ & $\begin{array}{l}0,05 \\
0,33\end{array}$ & $\begin{array}{l}6 \\
4\end{array}$ \\
\hline ecla & $\begin{array}{l}12 \\
13\end{array}$ & $\begin{array}{l}\text { BM6404 } \\
\text { TGLA23 }\end{array}$ & $\begin{array}{l}0,08 \\
0,05\end{array}$ & $\begin{array}{l}5 \\
4\end{array}$ \\
\hline impa & 28 & BMS2060 & 0,04 & 4 \\
\hline Igtr & $\begin{array}{c}2 \\
21 \\
27\end{array}$ & $\begin{array}{c}\text { ILSTS082 } \\
\text { BM8115 } \\
\text { INRAMTT183 }\end{array}$ & $\begin{array}{l}0,17 \\
0,10 \\
0,02\end{array}$ & $\begin{array}{l}2 \\
4 \\
2\end{array}$ \\
\hline hsac & $\begin{array}{c}5 \\
6 \\
13\end{array}$ & $\begin{array}{c}\text { BM 315 } \\
\text { BM 1329 } \\
\text { ABS10 }\end{array}$ & $\begin{array}{l}0,01 \\
0,24 \\
0,40\end{array}$ & $\begin{array}{l}4 \\
4 \\
2\end{array}$ \\
\hline ppoi & 11 & BMS1822 & 0,20 & 4 \\
\hline loba & $\begin{array}{c}5 \\
20\end{array}$ & $\begin{array}{c}\text { BM315 } \\
\text { TGLA126 }\end{array}$ & $\begin{array}{l}0,19 \\
0,37\end{array}$ & $\begin{array}{l}3 \\
4\end{array}$ \\
\hline laba & $\begin{array}{l}5 \\
6 \\
8\end{array}$ & $\begin{array}{c}\text { BM315 } \\
\text { DIK82 } \\
\text { BMS2847 }\end{array}$ & $\begin{array}{l}0,05 \\
0,32 \\
0,10\end{array}$ & $\begin{array}{l}4 \\
4 \\
5\end{array}$ \\
\hline inba & $\begin{array}{l}1 \\
13 \\
19\end{array}$ & $\begin{array}{l}\text { BM4307 } \\
\text { HUj } 616 \\
\text { ETH3 }\end{array}$ & $\begin{array}{l}0,18 \\
0,06 \\
0,23\end{array}$ & $\begin{array}{l}4 \\
3 \\
5\end{array}$ \\
\hline epta & 15 & BMS2684 & 0,31 & 4 \\
\hline
\end{tabular}

Pour les caractères de production et de qualité du lait, toutes les régions chromosomiques mentionnées dans différentes études sont retrouvées, en particulier les chromosomes 6, 14, 20 et 26 (Georges et al 1995, Spelman et al 1996, Vilkki et al 1997, Coppieters et al 1998, Ron et al 1998, Zhang et al 1998). Dans tous les cas, le nombre de familles informatives, c'est-à-dire avec le père hétérozygote pour le QTL, est relativement limité et toujours inférieur à 5 sur 14 . Ceci est attendu et s'explique par le fait que les allèles de QTL à effet important sont sans doute relativement rares. II est donc important de disposer d'un nombre suffisant de grandes familles et donc d'un dispositif de grande taille, seule une fraction de ce dispositif étant informative.

Les résultats concernant les autres caractères sont tout à fait originaux. On notera en particulier que des QTL sont trouvés pour pratiquement tous les caractères, y compris ceux peu héritables comme la résistance aux mammites (cell), la fertilité (fer), ou la qualité du sabot (epta). Comme le prévoit la théorie, on vérifie donc que le dispositif 'petites-filles' reste efficace même pour les caractères de faible héritabilité.

Le tableau 4 fournit la liste des QTL détectés avec un risque par analyse compris entre 0,4 et $1 \%$. Parmi l'ensemble des résultats des tableaux 3 et 4 , on attend environ 6 faux positifs. A nouveau, on retrouve des résultats déjà décrits dans différentes études, en particulier les chromosomes 3 et 7 pour les caractères de production laitière. On constate que certaines zones ont un effet sur plusieurs caractères simultanément (chromosomes 7 ou 14). Dans ce cas, les effets observés sont toujours dans le sens prédit par la corrélation génétique. Ainsi, quand un QTL affecte à la fois la quantité de lait et les taux, l'allèle qui accroît la quantité de lait est défavorable pour les taux.
Tableau 4. Liste des QTL détectés avec un risque compris entre 0,4 et $1 \%$ (6 faux positifs attendus pour l'ensemble des deux tableaux 3 et 4). $n$ : nombre de familles.

\begin{tabular}{|c|c|c|c|c|}
\hline Caractère & $\begin{array}{c}\text { Chromo } \\
\text {-some }\end{array}$ & $\begin{array}{c}\text { marqueur } \\
\text { proche }\end{array}$ & $\begin{array}{c}\text { risque } \\
(\%)\end{array}$ & $\mathrm{n}$ \\
\hline lait & 11 & INRABER162 & 0,82 & 2 \\
\hline $\mathrm{mp}$ & 7 & INRABER192 & 0,75 & 3 \\
& 19 & ETH3 & 0,83 & 4 \\
\hline tp & 3 & ILSTS096 & 0,98 & 5 \\
& 18 & ABS13 & 0,82 & 2 \\
cell & 20 & TGLA126 & 0,72 & 3 \\
\hline vtr & 10 & DIK20 & 0,93 & 3 \\
& 6 & BM2320 & 0,82 & 4 \\
& 8 & BMS678 & 0,76 & 4 \\
\hline eqaa & 13 & ABS10 & 0,81 & 3 \\
& 14 & UWCA9 & 0,57 & 4 \\
& 20 & CSSM066 & 0,89 & 2 \\
\hline ecla & 2 & TGLA126 & 0,76 & 4 \\
\hline ecav & 11 & IDVM042 & 0,52 & 3 \\
& 17 & BM1862 & 0,83 & 2 \\
\hline Igtr & 28 & IDVGA8 & 0,84 & 4 \\
\hline ppoi & 28 & IDVGA8 & 0,91 & 2 \\
& 2 & CSSM042 & 0,77 & 1 \\
& 2 & BMS1967 & 0,74 & 4 \\
\hline laba & 24 & ILSTS031 & 0,57 & 3 \\
\hline inba & 11 & BM1225 & 0,41 & 4 \\
\hline epta & 17 & BMS2208 & 0,70 & 3 \\
\hline fert & 7 & BMS499 & 0,53 & 2 \\
\hline & 7 & BMM741 & 0,83 & 1 \\
\hline & & INRA073 & 0,57 & 4 \\
& & & 0,51 & 4 \\
\hline
\end{tabular}

Les effets mis en évidence sont généralement compris entre 0,6 et 1 écart type génétique d'effet de substitution allélique. Autrement dit, l'écart entre deux groupes de filles ayant reçu l'un ou l'autre allèle paternel d'un QTL donné est de l'ordre de 300 à $500 \mathrm{~kg}$ de lait, 1,5 à $2 \mathrm{~g} / \mathrm{l}$ de taux butyreux, 0,6 à 1 point de morphologie, ou 3 à 5 points de taux de réussite à l'insémination. Ces effets sont donc importants et utilisables en sélection.

Enfin, le tableau 5, de loin le plus fourni, donne tous les résultats significatifs avec un risque d'erreur compris entre 1 et $4 \%$ Parmi l'ensemble des 135 résultats des tableaux 3, 4 et 5, on attend environ 28 faux positifs et donc plus de 100 vrais QTL. Contrairement aux résultats précédents, I'utilisation pratique de cette série de résultats demande une étape de confirmation. Cette étape peut être réalisée à partir de données indépendantes, soit françaises, ce qui demanderait un effort important, soit étrangères, à partir de collaborations ou tout simplement de la bibliographie. Par exemple, on peut être très confiant sur le QTL de matière protéique détecté sur le chromosome 17, déjà cité par Zhang et al (1998).

Cet article ne présente que les résultats obtenus à partir des transmissions paternelles. Le dispositif permet de tirer plus d'information. Les mères ayant un nombre très limité de descendants, il n'est pas possible d'analyser les ségrégations intra mere. Cependant, les mères peuvent apporter une information supplémentaire, de deux façons complémentaires. D'une part, les mères sont parfois demisœurs de père. On constate donc que les fils se regroupent en grosses familles de petits-fils issus d'un même grand-père maternel. Ainsi, Valiant, B ell, Rotate, Mark, Ned Boy, Secret, Cleitus en race Holstein, Newlook et Martien en race Montbéliarde, disposent d'un nombre important de petits-fils typés. Bien que la méthodologie soit un peu plus complexe et que le dispositif soit moins puissant (car seuls la moitié des petits-fils reçoivent un allèle grand-paternel à un locus donné), l'analyse intra 
grand-père constitue une source de validation indépendante et élégante de l'analyse intra-père. Ce travail est en cours. La deuxième méthode de validation, plus délicate à mettre en œuvre compte tenu de la densité de marquage assez faible, vise à rechercher un déséquilibre de liaison entre allèle marqueur et allèle de QTL, en supposant que l'origine du déséquilibre est très récente et qu'il n'est pas encore détruit par les recombinaisons au cours des générations. On peut supposer cependant que ce type d'analyse nécessite des marqueurs et des typages supplémentaires.

Tableau 5. Liste des 77 QTL détectés avec un risque compris entre 1 et $4 \%$ (28 faux positifs attendus pour l'ensemble des trois tableaux 3, 4 et 5).

\begin{tabular}{|l|l|}
\hline Caractères & \multicolumn{1}{|c|}{ Chromosomes (risque p, en \%) } \\
\hline lait & $7(2,3), 19(3,1), 26(2,1)$ \\
\hline $\mathrm{mp}$ & $17(3,5), 22(2,7)$ \\
\hline tb & $7(1,6), 15(1,8), 19(1,6), 22(2,3)$ \\
\hline tp & $15(2,2)$ \\
\hline cell & $9(1,3), 21(1,1), 23(1,7)$ \\
\hline fert & $5(3,5), 10(2,5), 20(1,5), 21(1,6)$ \\
\hline vtr & $5(3,7), 19(1,7), 21(3,2), 22(1,4)$ \\
\hline psil & $10(3,3), 11(1,1), 20(1,2), 25(1,6)$ \\
\hline dplj & $4(2,6), 6(2,6), 9(1,1), 10(3,4), 13(2,3), 20(1,1)$, \\
& $21(1,2)$ \\
\hline eqaa & $1(1,8), 16(4,0)$ \\
\hline ecla & $4(1,4), 10(2,2), 18(2,3), 21(1,8)$ \\
\hline ecav & $6(2,4), 9(2,4), 15(4,0), 24(2,7)$ \\
\hline impa & $2(2,3), 8(2,1), 9(2,3), 11(2,1), 15(1,3), 24(2,7)$ \\
\hline hata & $5(1,4), 6(1,4), 9(1,4)$ \\
\hline Igtr & $6(2,9), 8(1,6), 13(1,9)$ \\
\hline hsac & $1(3,9), 2(1,6), 11(1,4)$ \\
\hline ppoi & $1(2,0), 3(3,8), 5(1,2), 6(3,5), 8(3,7), 16(4,0)$, \\
\hline & $20(2,7)$ \\
\hline Ioba & $1(2,6), 11(3,0), 26(3,6), 28(1,8)$ \\
\hline laba & $11(2,3)$ \\
\hline inba & $5(2,9), 8(1,3), 14(3,2), 28(1,4)$ \\
\hline anja & $9(4,0)$ \\
\hline epta & $9(1,8), 18(2,3), 24(3,7)$ \\
\hline
\end{tabular}

\section{Conclusion et perspectives}

Cette étude à grande échelle a permis de disséquer la variabilité génétique des caractères sélectionnés dans trois races bovines laitières. Elle confirme la plupart des résultats connus à ce jour et fournit de nombreux résultats originaux, en particulier sur la morphologie et la fertilité. Ce travail doit maintenant être complété pour confirmer certains QTL qui, en l'état actuel des connaissances, peuvent être des faux positifs. Pour les QTL les plus intéressants, il est sou- haitable d'affiner la localisation avant, peut-être, d'envisager la caractérisation complète et l'identification des gènes impliqués. Les techniques d'identité par descendance, qui recherchent le plus petit haplotype portant le QTL et conservé entre familles, voire entre races, et, plus généralement, les techniques basées sur l'utilisation d'un éventuel déséquilibre de liaison dans la population, sont des méthodes de choix pour réduire l'intervalle de confiance de la position d'un QTL Ces méthodes demandent cependant un important travail de génotypage supplémentaire.

Les effets de QTL détectés sont importants et sont mis en évidence sur les populations actuellement en sélection. Ils sont donc directement utilisables en sélection assistée par marqueurs. L'imprécision de la localisation n'est pas un handicap insurmontable, compte tenu de la taille des segments transmis d'une génération à l'autre. La principale difficulté réside dans l'équilibre de liaison supposé intra population, qui limite considérablement l'efficacité de la sélection. En effet, une association entre un allèle marqueur et un allèle QTL détecté dans une famille n'est pas général isable à l'ensemble de la population. Deux solutions sont envisageables. La plus simple est de restreindre I'utilisation des marqueurs à une sélection intra famille de parents hétérozygotes au(x) QTL(s), les produits ayant reçu l'allèle favorable étant sélectionnés. Une solution plus complexe consiste à estimer la valeur génétique au QTL (et pour le reste du génome) de l'ensemble des candidats reproducteurs pour pratiquer une sélection au sein de la population globale. Cette approche revient à prédire le génotype des reproducteurs homozygotes, ce qui est réalisable soit avec des méthodes statistiques grâce à la connexion entre les pedigrees, soit en recherchant les déséquilibres de liaison qui existent inévitablement chez les bovins laitiers, compte tenu de l'impact récent d'un nombre très limité de fondateurs. Des investissements méthodologiques et pratiques importants sont nécessaires dans ce domaine pour valoriser rapidement les résultats obtenus.

\section{Remerciements}

Ce programme a été financé par le Département de Génétique Animale de I'INRA, par le Groupe d'E tude et de Recherche sur les Génomes et par I'UNCEIA et ses coopératives membres.

\section{Références}

Boichard D., Maignel L., Verrier E., 1996. Analyse généalogique des races bovines laitières françaises. INRA Productions Animales, 9, 323-335.

Boichard D., Le Roy P., Levéziel H., Elsen J.M., 1998. Utilisation des marqueurs moléculaires en génétique animale. INRA Productions Animales, 11, 67-80.

Churchill G.A., Doerge R.W., 1994. Empirical threshold values for quantitative trait mapping. Genetics, 138, 963-971.

Coppieters W., Riquet J ., Arranz I .J ., et al, 1998. A QTL with major effect on milk yield and composition maps to bovine chromosome 14. Mammalian Genome, 9, 540-544.
Elsen J .M., Mangin B., Goffinet B., et al, 1999. Alternative models for QTL detection in livestock. I General introduction. Genetics Selection Evolution, 31, 213-224.

Georges M., Nielsen D., Makinnon M., et al, 1995. Mapping quantitative trait loci controlling milk production in dairy cattle by exploiting progeny testing. Genetics, 139, 907-920.

Goffinet B., Boichard D., Le Roy P., et al. 1999. Alternative models for QTL detection in livestock. III Assumptions behind the model. Genetics Selection Evolution, 31, 341-350.

Ron M., Heyen D.W., Weller J .I., et al, 1998. Detection and analysis of a locus affecting milk concentration in the US and Israeli 
dairy cattle populations. Proceedings of the 6th World Congress on Genetics Applied to Livestock Production, 26, 422-425.

Spelman R.J., Coppieters W., Karim L., et al, 1996 Quantitative trait loci analysis for five milk production traits on chromosome six in the Dutch Holstein-Friesian population. Genetics, 144, 1799-1808.

Vaiman D., 2000. Etablissement des cartes génétiques. INRA Productions Animales, numéro hors série "Génétique moléculaire : principes et application aux populations animales », 73-78.
Vilkki H.J., de Konning D.J ., Elo K., et al, 1997. Multiple marker mapping of quantitative trait loci of Finnish dairy cattle by regression. J ournal of Dairy Science, 80, 198-204.

Weller J .I., Kashi Y., Soller M., 1990. Power of daughter and granddaughter designs for determining linkage between marker loci and quantitative trait loci. J ournal of Dairy Science, 73, 2525-2537.

Zhang Q., Boichard D., Hoeschele I., et al, 1998. Mapping quantitative trait loci for milk production and health of dairy cattle in a large outbred pedigree. Genetics, 149, 1959-1973. 